\title{
Wireless Monitoring and Control System Via Android Tablet PC
}

\author{
Zhen Huang \\ School of Automation \\ Wuhan University of Technology \\ Wuhan, China \\ h-zhen@163.com
}

\author{
Yunhan Lu \\ School of Automation \\ Wuhan University of Technology \\ Wuhan, China \\ luyunhan@yahoo.com.cn
}

\begin{abstract}
Typical industrial remote monitoring and control systems are usually based on PC, but it can hardly be applied to fields that need mobile communication. Along with the developing of wireless communication technology and mobile device, using mobile devices to realize wireless remote monitoring and control becomes possible and has vast development space. But the generally wireless remote monitoring and control can hardly realize a real time and continues control. In this paper we design a wireless monitoring and control system base on Android tablet PC for OGSTSS (oil and gas storage and transportation simulation system) to realize a real time and continues control.
\end{abstract} socket

Keywords-monitoring and control system;WLAN; Android;

\section{INTRODUCTION}

Typical industrial remote monitoring and control system use a hierarchical structure. It usually has three layers: filed layer, control layer and management layer. Filed layer deal with kinds of sensors and actuators, field bus such as PROFIBUS, MODBUS and RS485,are adopted. Control layer consists of local controllers such as kinds of PCs or embedded panels. Management layer provide premium service such remote monitoring and control, data recording service and web service. Both control and management layer are base on Ethernet network to communication with each other[1]

This kind of monitoring and control system generally uses PC as the monitoring and control point in system, but it can hardly be applied to fields that need mobile communication, such logistics management, maintenance of machines and mobile monitoring and control.

Along with the developing and popularization of wireless communication technology and mobile devices. Using mobile devices to realize wireless remote monitoring and control becomes possible and has vast development space[24].

At present, general wireless remote monitoring and control system has two ways to realize. One method is using mobile device (generally are mobile phones) which support connecting to IP (internet protocol) networks via mobile communication networks. Users can use this achieving a remote monitoring and control system, but the wireless data packet system is too expensive. Another method is using SMS (Short Message Service). Users can achieve a remote monitoring and control system too. But this method is inadequate for continuous control[5]. Because of the pool performance of mobile phone, such as the small screen size, and support few communication mode it can hardly be called as a real time control system.

In this paper we solve the limitation of the existing method, by using WLAN (wireless local area network) technology and Android tablet PC.

In Table I we can see the wireless communication technologies that wildly used. They can be dived into two sorts: short distance and long distance wireless communication technologies.

In short distance communication technologies, Wi-Fi has a high communication speed and suitable communication distance. Bluetooch, Zigbee and DSRC (dedicated short range communications) have low communication speed or very short communication distance. In long distance communication technologies. GSM (global system of mobile communication), GPRS (general packet radio service) and digital broadcasting station have a low communication speed. 3G and WIMAX (worldwide interoperability for microwave access) have high communication speed. GSM, GPRS, 3G, WIMAX are commercial communication service, have high use fee. Digital broadcasting station need set up dedicated communication line. so in this paper we use $\mathrm{Wi}-\mathrm{Fi}$ as the wireless communication network.

$\mathrm{Wi}-\mathrm{Fi}$ is a popular name for the wireless Ethernet 802.11b standard for WLAN. The Wi-Fi is a well developed technology which can be used for remote monitoring and control[4].The generation of WLAN support up to 54Mbps data rates within $100 \mathrm{~m}$ of the base station.

TABLE I. COMMON COMMUNICATION TECHNOLOGIES

\begin{tabular}{|c|c|c|}
\hline Name & $\begin{array}{c}\text { Communication } \\
\text { speed }\end{array}$ & $\begin{array}{c}\text { Communication } \\
\text { distance }\end{array}$ \\
\hline Wi-Fi & $54 \mathrm{Mbps}$ & $100 \mathrm{~m}$ \\
\hline Bluetooch & $1 \mathrm{Mbps}$ & $10 \mathrm{~m}$ \\
\hline Zigbee & $<250 \mathrm{Kbps}$ & $100-1200 \mathrm{~m}$ \\
\hline DSRC & $250 \mathrm{Kbps}-$ & $10 \mathrm{~m}$ \\
\hline GSM & $500 \mathrm{Kbps}$ & $>35 \mathrm{Km}$ \\
\hline GPRS & $1114 \mathrm{Kbps}$ & $>35 \mathrm{Km}$ \\
\hline 3G & $2 \mathrm{Mbps}$ & $>35 \mathrm{Km}$ \\
\hline WIMAX & $45 \mathrm{Mbps}$ & $50 \mathrm{Km}$ \\
\hline Digital broadcasting station & $9600 \mathrm{Kbps}$ & $30 \mathrm{Km}$ \\
\hline
\end{tabular}


TABLE II. COMMON MOBILE DEVICE

\begin{tabular}{|c|c|c|}
\hline Name & Describe & $\begin{array}{c}\text { Communication } \\
\text { mode }\end{array}$ \\
\hline PDA & $\begin{array}{c}\text { Wireless communication, } \\
\text { multi-media, 2-3inchs screen }\end{array}$ & Bluetooth, Wi-Fi \\
\hline $\begin{array}{c}\text { Smart } \\
\text { phone }\end{array}$ & $\begin{array}{c}\text { Wireless communication, } \\
\text { voice communication, multi- } \\
\text { media, 2-5inchs screen }\end{array}$ & $\begin{array}{c}\text { GSM, GPRS, 3G, } \\
\text { Bluetooth, Wi-Fi }\end{array}$ \\
\hline Tablet PC & $\begin{array}{c}\text { Wireless communication, } \\
\text { multi-media, 5-12inchs screen }\end{array}$ & 3G, Bluetooth, Wi-Fi \\
\hline MID & $\begin{array}{c}\text { Wireless communication, } \\
\text { multi-media, 4-7inchs screen }\end{array}$ & 3G, Bluetooth, Wi-Fi \\
\hline UMPC & $\begin{array}{c}\text { Wireless communication, } \\
\text { multi-media, 7-12inchs screen }\end{array}$ & 3G, Bluetooth, Wi-Fi \\
\hline
\end{tabular}

Table II is the common mobile device used wildly. Smart phone and Tablet PC are the most popular mobile devices among those devices. Smart phone have the best portability and communication mode, but tablet PC has higher performance than most smart phones, bigger screen size, and better user experience. The tablet PC is suitable for the wireless monitoring and control system.

Android OS (operating system)is the most popular mobile device OS around the world. It has a stronger action experience and it is a real open source OS, it provides the tools and APIs (application program interface ) necessary to develop application on the Android platform.

We will design a wireless monitoring and control system based on Android tablet PC and Wi-Fi networks. This system can realize a low price, real time, continuous controlling monitoring and control.

This paper is organized as follows. In Section II, we introduce the structure of the wireless monitoring and control system. SectionIII, the design of wireless communication software. SectionIV, the implement of wireless monitoring and control system, and the conclusion.

\section{GENERAL DESIGN OF SYSTEM}

\section{A. oil and gas storage and transportation simulation system}

The wireless monitoring and control system we designed is a part of an oil and gas storage and transportation simulation system (OGSTSS), which is a scale model of an actual oil and gas storage and transport system. This scale model can demonstrate the operating mechanism of an actual system. The different between the scale model and actual system is that the former uses LED (light emitting diode) lamps to stand for the equipment in the actual system such as pumps, valves, pipelines. The OGSTSS has 5 parts: car station system, pump room, tank field, train station system and dock area. All digital I/O signals sum to 1164 point. This OGSTSS can automatically demonstrates the typical process of oil and gas storage and transportation system, such as car load, train load, oil tanker load, transit operation of oil tanks, and support manual operation of those process. The monitoring and control system of OGSTSS can apply to an actual oil and gas storage and transportation system, by set up external devices such as sensors and actuators.

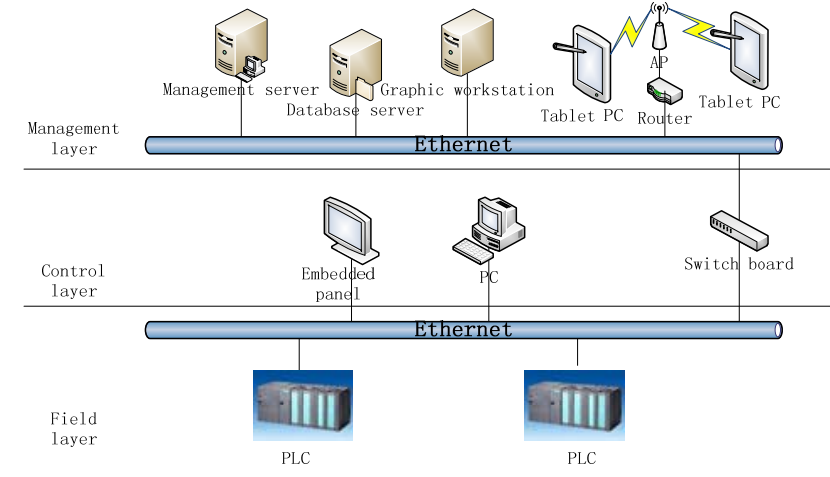

Figure1. The framework of whole system

\section{B. The design of monitoring and control system}

The monitoring and control system of OGSTSS with hierarchical structure is made up of field layer, control layer, management layer(see Figure 1).

The field layer is mainly make up of PLC controller. PLC controller which is used in many industries and machines is the base of whole system. It carries out the control commands such as controlling the start and stop of pumps or valves, and monitors the data such as machine's running status independent. PLC controller can send running status and receive control command from the PC station, embedded panel and Tablet PC. Field layer uses a field bus structure. PLC controller can connect to field bus with conversion gateway or liner. With the using of field bus structure, the OGSTSS can set up external devices if them needed.

The control layer is made up of PC station and embedded panel. PC station is a remote monitoring and control point in the system. It can monitor the running status received from PLC controller, and send the control command to change the running status of field device. At the same time PC station is a transfer station between PLC controller and tablet PC. It receives running status from PLC controller then sends to tablet PC, and it also sends control commands that come from tablet PC to PLC controller. Embedded panel has the same function, but with a higher control permission as PC station. Because embedded panel is a local monitoring and control point in the system.

The management layer is made up of management server, database server, graphic workstation and Tablet PC. Management server can realize the function of PC station and embedded panel. Data base server can store the system operation data. The graphic workstation can demonstrates the work process of OGSTSS with a 3D model way.

Tablet PC can monitor the field device's running status at real time, and send control commands to imply a continuous control. It is the most significant thing that user can do those operation at anywhere near the wireless AP (access point).

\section{Communication model design}

In OGSTSS Ethernet is adopted, especially in the field layer Ethernet is applied for field bus. Ethernet is the most wildly used LAN (local area network) technologies in office 
automation field, and is more and more accept by industry automation field.

\begin{tabular}{|c|}
\hline $\begin{array}{c}\text { PC } \\
\text { server }\end{array}$ \\
\hline OPC client \\
\hline $\begin{array}{c}\text { Ethernet } \\
\perp\end{array}$ \\
\hline OPC server \\
\hline PLC \\
controller \\
\hline
\end{tabular}

Figure 2. communication between PC server and PLC controller

We use OPC (object linking and embedding (OLE) for process control) protocol which provides a communication solution between hardware and software[6], and was wildly used in industry automation field, transfer data between control layer and field layer. The communication model is showed as Figure 2.

To realize the wireless monitoring and control via tablet PC we adopt Client/Server (C/S) model. In this C/S model, tablet PC is the client and PC station is the server. Tablet PC communication with PC station and then PC station communication with PLC controller. This structure can realize an indirectly communicate between tablet PC and PLC controller. Using this structure, tablet PC can communication with PLC controller without care about the communication interface between the tablet PC and PLC controller. Tablet PC connects with PC station with Wi-Fi.

\section{WIRELESS COMMUNICATION SOFTWARE DESIGN}

Tablet PC and PC station built a WLAN which support TCP/IP protocol. TCP/IP protocol is the most wildly used communication protocol in LAN. It can provide two protocols-TCP (transport control protocol) and UDP (user datagram protocol)- in transport layer. The communication between Tablet PC and PC station can be realized by socket communication technology. The socket model is showed as figure 3 .

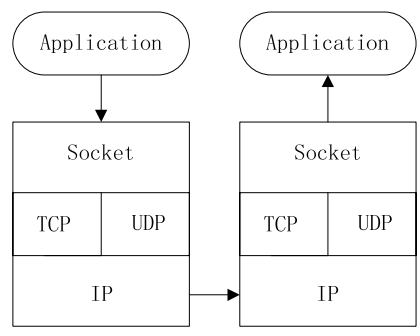

Figure 3. socket model

A socket is a communication connection point you can name and address in a network. There are two kinds of sockets, connection-oriented and connectionless. The TCP socket and UDP socket are two primary types of sockets to provide transport-layer network services. The TCP socket is connection-oriented mode, and the UDP socket is the connectionless mode. The TCP socket provides a reliable connection-oriented service and UDP socket provides a unreliable connectionless service. In view of the reliability requirements of the monitoring and control system, in this system, we adopt TCP socket.

TCP socket is based on C/S model. Here is the working principle of the TCP socket: firstly, the client invokes socket function to establish a socket. Then, invokes bind function to bind socket with local machine address and local port number. At the same time, the server invokes listen function to monitor in corresponding socket. When connection function receives a connection request, accept function will be generate a new socket. When the connection build up between client and server, we can use write function and read function to deal with data. By this way sending and receiving between client and server can be realized. Finally the socket is closed. The flow of socket is showed as figure 4 .

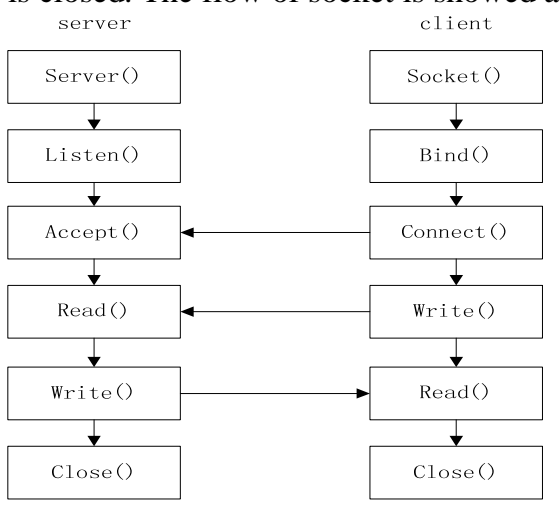

Figure 4. socket programming

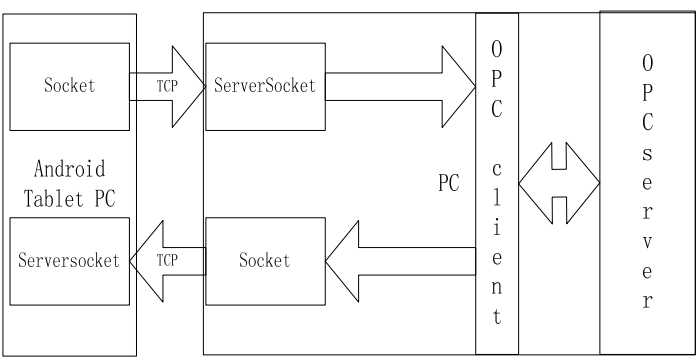

Figure.5 logic framework of the communication software

Figure.5 shows the logic framework of the communication software. Here we use a multiple-threads architecture. There are two threads - send thread and receive threads, and both of them are running the socket programs.

In send thread PC station is the socket server and the tablet PC is the socket client. This thread is used for send control command from tablet PC to PC station. The flow of this thread: when user give a control command the client program will invoke the socket communication, and the server program will receive the request of client program and built a socket connect. Then the control command is wrote in the output stream and sent to socket server program. When the control command is received, PC station will invoke the OPC service to send the control command to PLC controller, and PLC controller will carry out this command.

In receive thread tablet PC is the socket server and the PC station is the socket client. This thread is used for receive 
field device status from PC station to tablet PC. The flow of this thread: when PLC controller collects the changed status of field device, OPC service will send the message to socket client. The client program will invoke the socket communication as in send thread, and finally the user can monitor the field status on the tablet PC. Furthermore the receive thread has a higher priority level than send thread.

\section{IMPLEMENTION AND CONCLUSION}

We use a Motorola xoom mz606 tablet PC as the client, which powered Android 3.0 OS, a windows XP PC as the server. The tablet PC connects to PC through a wireless router.

We develop the client program on tablet PC by Java language, and develop the server program on PC by VB language, which can realize the socket server and OPC client function. Then we use a KEPServerEx V4.0 software as the OPC server and simulates the data source to test the tablet client program. The implementation of client program is showed as figure 6 .

In this paper, we design a wireless monitoring and control system for OGSTSS. Using Android tablet PC and WLAN technology, we can realize a real time and continues control. This kind of monitoring and control system has vast development space. Now this system can only realize the signal client mode and simple monitoring and control function, but in future, it can realize more senior function such as multi client, better UI(user interface) and regular function of monitoring and control system.

\section{REFERENCES}

[1] S. Chunshan, et al., "Key Design of Control-Monitoring System of Steady High Magnetic Field Facility," in Computational Intelligence and Industrial Application, 2008. PACIIA '08. Pacific-Asia Workshop on, 2008, pp. 81-85.
[2] M. Maaser and S. Ortmann, "Remote medical treatment at home using the Java Mobile Sensor API," in GLOBECOM Workshops (GC Wkshps), 2010 IEEE, 2010, pp. 2039-2043.

[3] L. Yonggang, et al., "Production Monitoring System of Coning, Drawing and Twisting Workshop Based on LAN," in Information Engineering, 2009. ICIE '09. WASE International Conference on, 2009, pp. 333-336.

[4] J. J. Zhou, et al., "Remote Monitoring and Automatic Navigation System for Agricultural Vehicles Based on WLAN," in 2008 4th International Conference on Wireless Communications, Networking and Mobile Computing, Vols 1-31, ed, 2008, pp. 2991-2994.

[5] H. Haeil, et al., "PC application remote control via mobile phone," in Control Automation and Systems (ICCAS), 2010 International Conference on, 2010, pp. 2290-2294.

[6] S. Mai and Y. Myeong-Jae, "A study on OPC specifications: Perspective and challenges," in Strategic Technology (IFOST), 2010 International Forum on, 2010, pp. 193-197.

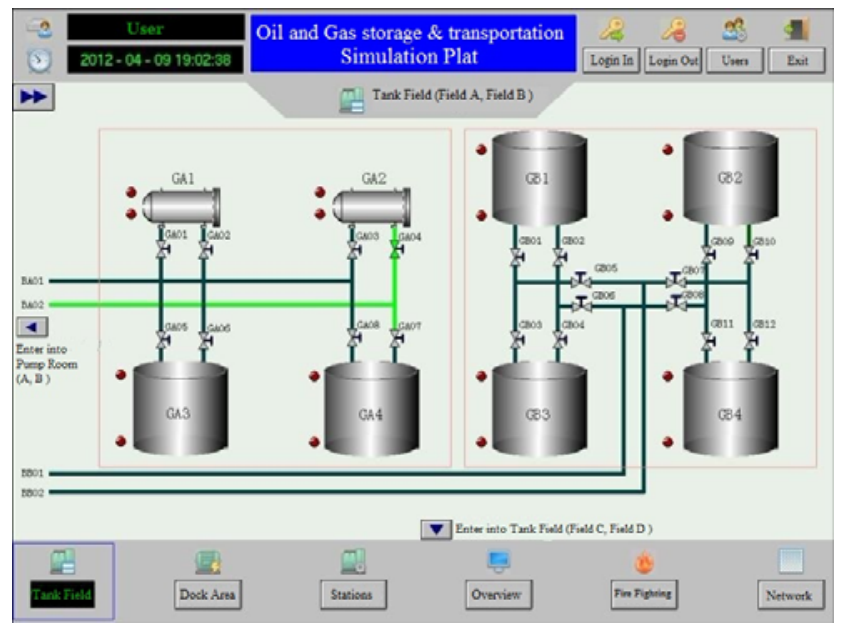

Figure.6 Implementation of client program 